\title{
Germanica
}

\section{« Manche sagen, ich bin irre... » Anmerkungen zu Monika Marons Roman Die Überläuferin}

"Manche sagen, ich bin irre..." Remarques à propos du roman Die

Überläuferin / La transfuge de Monika Maron

\section{Alice Bolterauer}

\section{OpenEdition}

\section{Journals}

Édition électronique

URL : http://journals.openedition.org/germanica/1852

DOI : 10.4000/germanica.1852

ISSN : 2107-0784

\section{Éditeur}

Université de Lille

\section{Édition imprimée}

Date de publication : 30 juin 2003

Pagination : 123-135

ISBN : 9782913857117

ISSN : 0984-2632

Référence électronique

Alice Bolterauer, « « Manche sagen, ich bin irre... » Anmerkungen zu Monika Marons Roman Die Überläuferin », Germanica [Online], 32 | 2003, Online erschienen am: 10 Dezember 2012, abgerufen am 06 Oktober 2020. URL : http://journals.openedition.org/germanica/1852 ; DOI : https://doi.org/ 10.4000/germanica. 1852

Ce document a été généré automatiquement le 6 octobre 2020.

(C) Tous droits réservés 


\title{
"Manche sagen, ich bin irre...» Anmerkungen zu Monika Marons Roman Die Überläuferin
}

\author{
"Manche sagen, ich bin irre... » Remarques à propos du roman Die \\ Überläuferin / La transfuge de Monika Maron
}

Alice Bolterauer

«Ce n'est pas la crainte de la folie qui nous
forcera à laisser en berne le drapeau de
l'imagination. »
André Breton, Manifeste du surréalisme

1.

«Wenn die Welt irre ist, liegt im Irrsinn der Sinn ${ }^{1}$.» Die das sagt, ist die supponierte Ich-Erzählerin des Romans von Monika Maron Die Überläuferin (1986), eines Textes, der - und darin ist sein Titel programmatisch - alle Grenzen in Frage stellt, sie überschreitet oder einfach umwirft : Grenzen des Guten, des Politisch-Korrekten, des Angesehenen, des Vernünftigen, des Erzählens. Denn darum, gegen die Grenzen der instrumentellen Vernunft - sei es in politischer, existentiell-privater oder ästhetischer Hinsicht - anzukämpfen, über sie hinwegzufegen im stürmischen Entwurf einer grenzenlosen, an die Tradition des Surrealismus ebenso anknüpfenden wie das Aufbegehren aller Friedens- und Ökobewegungen weiterschreibenden Phantasterei, eben darum geht es in diesem Buch, das so mitreißt, wie es selbst mitgerissen scheint vom Sog eines nicht enden wollenden Hinabtauchens in die Welt der imaginierten Einsichten. Nein, nicht von der Hereinholung des Wahnsinnigen, des Irrsinnigen, des Unvernünftigen in unsere Welt des Sinns, der Normalität und der Vernunft ist hier die Rede, nicht von der Rehabilitation des verdrängten Anderen oder der Aktivierung des unterdrückten Fremden, sondern von der Explosivität einer immer bereits vorhandenen, bloß zu aktivierenden Welt des Surrealen. Nicht der emanzipatorische 
Impetus, zu retten, was einer rationalen Unterdrückungsmaschinerie zum Opfer fiel, bestimmt den Roman, sondern die Lust an der Überschreitung vernünftiger Ordnungszwänge, um - und dafür könnte in der Tat der literarische Text der prädestinierte Ort sein - so ein Spiel zu inszenieren um mögliche Lebensentwürfe, Identitätskonstruktionen, Weltbilder.

Die Potentialität des Irreseins, die hier nicht im Sinne von Foucault als Einspruch gegen die Ausschließung des Devianten zu sehen ist ${ }^{2}$ - schließlich befinden wir uns in einer Zeit der Postismen, der Wolfgang Welsch den Verlust eines «Standards von Normalität » attestiert ${ }^{3}$-, figuriert als notwendige Ergänzung einer selbstverschuldeten Einseitigkeit, die psychische Deformation meint und eine Einschränkung unserer Lebensmöglichkeiten bewirkt. In jeder Hinsicht.

\section{2.}

3 Natürlich ist die Entstehung des Romans «Die Überläuferin » vor dem Background zeitgenössischer DDR-Literatur der 70er und 80er Jahre zu sehen, der der Entwurf des Surrealen und Imaginären als Gegenentwurf zu der alles beherrschenden Dominanz instrumenteller Vernunft dient. Als die Sanktionen der offiziösen DDR-Kulturpolitik ab den späten 70er Jahren immer drastischere Formen annehmen, entsteht in der DDR eine phantastische Literatur, die "Einbildungen, Phantasmen, Fiktionen als eine zweite, andere Realität gegen die erste, verordnete setzt ${ }^{4}$. «Wenn Irmtraud Morgner, Fritz Rudolf Fries, aber auch Christa Wolf in diesen Jahren in der überbordenden Phantasie ihrer Texte der lange geschmähten Fiktionalität wieder ihr Recht einräumen, dann geschieht dies in dezidierter Abwehr einer von oben verordneten, von oben sanktionierten «Nützlichkeit» literarischer Texte, einer Instrumentalisierung zu pädagogischen und/oder ökonomischen Zwecken, der Literatur nur gerecht wird, wenn sie darauf verzichtet, sich selbst, d.h. ihren eigenen Gesetzen und Ansprüchen gerecht $\mathrm{zu}$ werden. Gegen diese nicht länger glaubhaft zu vermittelnde sinnhafte Einbindung literarischer Produktion in umgreifende ökonomische und soziale Transformationsprozesse richtet sich der Rekurs der DDR-Schriftsteller/innen jener Zeit auf die Sprengkraft scheinbar nutzlosen Fabulierens und Phantasierens. «Denn in einer historischen und gesellschaftlichen Lage ", heißt es etwa unter Bezug auf das Schreiben Irmtraud Morgners, "die durch ein Zuviel an System, an Ordnung, an Repression charakterisiert ist, muss die Kunst Ordnung zerstören, entordnen, im Wortsinn an-archisch sein ${ }^{5}$.»

4 Nirgends wird dieses Aufeinander-Bezogensein von sozial sanktionierter Ordnung und anarchisch-subjektiver Phantasie deutlicher als in jenen Szenen in Monika Marons erstem Roman «Flugasche » (1981), wo das imaginäre Potential der Protagonistin Josefa als Stein des Anstoßes, aber auch als Alternative zu den starren Verfahren, Denkund Sprachmustern offizieller Parteimitglieder und Parteifunktionäre fungiert. Wenn nämlich die Parteileitung von Josefas Betrieb tagt, um über die Parteitauglichkeit ihrer Kollegin zu debattieren, dann « rettet » Josefa nur der Blick aus dem Fenster auf einen Himmel, in dem die Wolken «flugverkehrswidrig, nur dem Wind gehorchend $»^{6}$ fliegen und so die Erinnerung an einen Tagtraum wachrufen, in dem sie mit Pawel, dem Freund, dessen Name jenen des geliebten Großvaters assoziiert, durch Wolkenberge geflogen ist. «Ich schließe die Augen und schwebe durch Finsternis, die silbern durch meine Lider zuckt ${ }^{7}$.» Der Realität eines sozialen Lebens, das nach den Gesetzen der 
instrumentellen Vernunft organisiert und legitimiert ist, kontrastiert Monika Maron das kreative Potential menschlicher Phantasie, das ebenso als subversiv wie als menschennotwendig dargestellt ist, so dass die Aktivierung individuell-subjektiver Imagination als in doppelter Hinsicht notwendig erscheint : als Korrektur eines sozialpolitischen Systems, das über der Bestärkung seiner machtstrategischen Potenzen auf die Befindlichkeit seiner Mitglieder vergessen hat, wie als Einforderung eines - wofür die zahlreichen Naturmetaphern in Monika Marons Werk stehen - ganzheitlichen Seins. Beides fließt zusammen und beide Aspekte bewirken eine verstärkte Hinwendung zur Sphäre des Surrealen und Imaginären, die die Nähe des Verrücktseins und Irreseins immer weniger scheut.

5 Im Unterschied zu dem frühen Roman «Flugasche », in dem die imaginierten Szenen als « Einsprengsel » in einem an sich eher « realistischen » Szenario fungieren, schlägt sich die Autorin in dem Roman "Die Überläuferin " ganz auf die Seite des Surrealen und Imaginären. Bereits die Vorstellung von Person und Handlung macht klar, dass hier auf « Realitätssinn » verzichtet wird. Nicht, dass nicht Realität erkennbar wäre weil auch das Surreale, was bereits Bert Brecht in der sog. Expressionismus-Debatte ${ }^{8}$ gegen ein reduziertes Realismus-Konzept ins Treffen geführt hatte, das Reale in sich enthalten kann -, aber die Eingangspassage stellt doch unmissverständlich fest, dass sie nicht als Widerspiegelung wiedererkennbarer Fakten verstanden werden will. Wenn sie, die Ich-Erzählerin, die letzten zwei Tage protokolliert, in denen sie weder Hunger noch Durst noch « ein aus der Nahrungsaufnahme resultierendes Bedürfnis » verspürt, noch jemals geschlafen hat (9), dann wird bereits der Anfang der Handlung auf eine Ebene der Potentialität und Irrealität gestellt, von der aus sich Realitäten erinnern oder imaginieren lassen, aber nicht länger als Realitäten in Erscheinung treten. Das ganze Buch ist ein Ringen um eine ebenso radikale wie präzise Surrealität, von der aus das Reale besser erkannt - und vielleicht auch verbessert - werden könnte.

6 In gewisser Weise findet hier eine Umkehrung der Perspektiven statt: Konnten in «Flugasche» die imaginierten Szenen, Tagvisionen und Träume als potentieller Einspruch gegen eine kastrierende, repressive Realität verstanden werden, so tauchen in der "Überläuferin», diesem fiktional-literarischen Einsatz einer surrealen Annäherung an die Wirklichkeit, die Vertreter der Ordnung und des Systems als mögliche Oppositionsvarianten auf, deren kritisches Potential aber schon dadurch geschmälert scheint, dass sie als Figuren einer surrealen Inszenierung, einer fiktiven, inszenierten Theateraufführung auftreten. Es sind die in die Handlung eingeschobenen "Zwischenspiele», in denen sich die Vertreter der öffentlichen Ordnung und Sicherheit präsentieren und ihre Ansprüche formulieren.

7 « Kommen wir zur Tagesordnung. » (35) Es ist der « Mann in der roten Uniform », der dies vorschlägt, der «mit den Fingerknöcheln auf den Tisch [klopft]» (35), der das inszenierte Schauspiel, das « Zimmertheater » (41), wie es die Ich-Erzählerin nennt, zu einer Parteitagung umfunktonieren will : «Punkt eins : Ordnung und Sicherheit. Punkt zwei : Sicherheit und Ordnung. Punkt drei : Ordenheit und Sicherung. » (35) Es ist ein inszeniertes, imaginiertes, als « Zimmertheater » auf die Privatheit seiner Inszenierung abgestelltes Tribunal über die Ordnungsuntauglichkeit der Protagonistin - «Die Anklage lautet: Unerlaubte Phantasie in Tateinheit mit Benutzung derselben im Wiederholungsfall»(170) -, eine von ihren Erfahrungen wie ihrem schlechten Gewissen gespeiste Vorstellung eines möglichen Zur-Rechenschaft-Gezogenwerdens, in dem die bekannten Sätze und Phrasen sozialer Verantwortlichkeit zitiert und zugleich 
ad absurdum geführt werden. Das ist etwa der Fall, wenn die monotone Beschwörung von "Ordnung " und "Sicherheit " zur sinnlosen Wortvermengung von "Ordenheit und Sicherung » wird, wenn der Streit um die gerechte Marx-Auslegung die Feuerwehr von ihrem Auftrag, Feuer zu löschen, befreit oder wenn in einem Atemzug die Referenz auf eine « Dienststelle » als « Sinn des Lebens » wie als mögliches Druckmittel figuriert.

Der Einspruch gegen die Berechtigung der "Ordnung », der Ordnung des Sozialen, des Politischen wie des Denkens - «Es geht um Ordnung und Sicherheit. Um die Sicherheit der Ordnung des Kopfes" (174), heißt es -, muss in einer Gesellschaft als staatsgefährdendes Verbrechen erscheinen, die nicht nur (wie westliche Demokratien dies tun) darauf hofft, dass jede/r ihren/seinen Platz in ihr finden möge, und die diejenigen, die dies nicht wollen oder können, als Außenseiter toleriert, sondern die die Brauchbarkeit jedes Einzelnen zum non plus ultra ihrer Legitimation und ihrer postulierten Überlegenheit macht; die die sozial-ökonomische Integration des Einzelnen - nicht nur seine potentielle, sondern seine konkret-reale - als Baustein einer kollektiven Ordnung und als deren Garanten versteht, wie sie umgekehrt in der praktisch unter Beweis gestellten Brauchbarkeit des Einzelnen die «Ordnung seines Kopfes » garantiert sieht. Die Emphase für das « Nutzlose », d.h. das Nicht-Brauchbare, muss einem solchen System (und natürlich ist dahinter immer das rigide System des SED-Staates gemeint) nicht nur als unnütz, subversiv, allzu kritisch, sondern in der Tat als Infragestellung des gesamten Staatsgefüges erscheinen und deswegen mit allen möglichen Mitteln - von denen im Roman «Die Überläuferin » von der verbalen Drohung bis zum Mord etliche benannt und virtuell erprobt werden - bekämpft werden. Dass damit die "Überläuferin» in Realitätsdingen zur politischen «Überläuferin » zu werden droht, ist von der zeitgenössischen DDR-Kritik durchaus richtig bemerkt worden.

\section{3.}

\footnotetext{
«Aber in jedem Menschen gibt es etwas, das sie nicht gebrauchen können, das Besondere, das Unberechenbare, Seele, Poesie, Musik, ich weiß keinen passenden Namen dafür, eben das, was niemand wissen konnte, ehe der Mensch geboren war. Dieses scheinbar nutzloseste Stück von dir mußt du finden und bewahren, das ist der Anfang deiner Biografie. » (51)
}

Was Martha, der Ich-Erzählerin alter ego, hier zitiert, sind die Lehren des Mathematikprofessors, des Chefs der Piraten - «die meisten Piraten waren Wissenschaftler, Maler oder Dichter»(50) -, die "den Nutzen des Nutzlosen zu erforschen " (50) sich vorgenommen haben und, weil finanzielle Unterstützung für dieses Vorhaben nicht $\mathrm{zu}$ erwarten ist, sich durch Piraterie finanzieren. Die Unverwechselbarkeit des Subjekts, eben seine Individualität, nicht in seiner Brauchbarkeit, in seiner sozial-ökonomischen Nützlichkeit zu suchen, sondern in seinen sich jeder rationalen Verwertbarkeit entziehenden kreativen, intuitiven, fiktionalen und animalischen Potenzen, darin liegt ein nicht unwesentlicher Impuls von Monika Marons Schreiben. Hierher gehört jene in den Texten Monika Marons immer wieder evozierte Nähe vor allem weiblicher Figuren zu Tieren und Tierischem, ihre Verwandlung in Tiere - seien es Vögel, Schlangen oder Äffinnen -, ihre fiktive Positionierung in animalisch-kreatürlichen Kontexten, in die etwa die Ich-Erzählerin von «Animal triste» (1996) zurückkehrt: "Ich bin eins von ihnen [von den sie umgebenden Tieren, A.B.], eine braunhaarige Äffin mit einer stumpfen Nase und langen 
Armen, die ich um meinen Tierleib schlinge $»^{9}$, um eine Ganzheit des Subjekts zu imaginieren, die realiter nicht mehr herstellbar ist.

Auch in dem Roman Die Überläuferin wird die Idee der Ganzheit des individuellen Subjekts nur als eine nostalgische oder als eine im utopischen Raum des fiktionalen Entwurfs vorstellbare evoziert - eine Evokation jedoch, die aus der Erfahrung eines defizitären Seins nach nur rationalen Ansprüchen und ökonomischen Gesichtspunkten sich als unverzichtbar erweist. Denn das, worauf eine nur an Effizienz und Markttauglichkeit sich orientierende moderne Gesellschaft das individuelle Ich reduziert : eine körperlose Kopfmasse, ein Roboter, ein gut funktionierender Klon, erweist sich weniger als finanztechnische Notwendigkeit, sondern vor allem als Verrat an sich selbst, an den eigenen Fähigkeiten und Reichtümern. Dass diese inneren, verdrängten, nicht gebrauchten Reichtümer und Fähigkeiten nur um den Preis der "Verrückung» der Grenzen sozialer, politischer und psychischer Ordnung wiederzubeleben, $\mathrm{zu}$ erwecken, $\mathrm{zu}$ aktivieren sind - ohne dass deswegen eine neue Ganzheit restituierbar wäre -, das macht Monika Marons Text eindeutig klar. Wenn die moderne Gesellschaft nur unseren «Kopf», sprich unsere ökonomische und arbeitsstrategische Rationalität benötigt und alles andere, was unser Leben und unser Ich-Sein ausmacht - Seele und Körper etwa -, ausspart, wenn sie unter dem Postulat der Aufhebung kapitalistischer Entfremdung neue Formen der Entfremdung produziert und favorisiert, dann muss die Rückbesinnung auf das «Andere » unseres rationalen Ichs - um mit Hartmut und Gernot Böhme zu sprechen ${ }^{10}$ - auch als Einspruch gegen die Rationalitätsstrukturen moderner Gesellschaft, muss als Abweichung, Ver-rückung, als Anders-Sinn, Wahn-Sinn empfunden und inszeniert werden. Martha und Clairchen diese zwei Alternativentwürfe des erzählenden Ichs - bewegen sich ständig an der Grenze des Zulässigen, des Legalen und des Normalen. So wie sich Martha in einem Bereich permanenter Gesetzesbrüche aufhält : sie lügt, stiehlt, erfindet Geschichten, was sie in den Geruch des «Irreseins » bringt, so provoziert Clairchen in ihrer alles überbordenden Körperfülle und in ihrer endlosen Sentimentalität die abfällige Missachtung durch die Gesellschaft, die sie schließlich in den Selbstmord treibt. Während sich Martha in ihrer Unbekümmertheit um Vorschriften und Konventionen zusehends befreit, d.h. für eine Freiheit des Denkens und Handelns steht, die sie entweder ins Gefängnis oder aber nach Amerika bringt, konfrontiert Clairchen, die sich in der belaubten Krone eines Kastanienbaums erhängt, ihre Umwelt mit der schockierenden Realität ihrer naiven Körperlichkeit. Beide Varianten inszenierter Abweichung von den geforderten und zulässigen Formen anerkannter Rationalität werden von der Umwelt mit den Stigmata des « Nicht-Normalen », des "Verrückten » und/oder des «Gefährlichen » bedacht. «Darum ham ooch alle so ville Angst vor den Dicken, instinktiv, weil bei denen dit Verhältnis von Hirn- und Körperfleischzellen inadäquat is, verstehste, inadäquat » (120), erkennt Clairchen. "Manche sagen, ich bin irre » (29), weiß auch Martha, deren Vater sie beispielsweise «schlicht für übergeschnappt » (53) hält.

11 In der Provokation, die Martha und Clairchen für ihre Umwelt bedeuten, liegt auch die Faszination der beiden Figuren für die Ich-Erzählerin Rosalind. « Noch heute halte ich Menschen, die stehlen und lügen können, für freiere Geschöpfe als die übrigen, die es nicht können. » (48) In der Beschäftigung mit den beiden Frauen eröffnen sich auch für die kühle Rosalind, dritte im Bunde und wohl die rationalste von den dreien - « Ich bin jetzt nur noch ein Kopf » (115), sagt sie -, neue Wege des Denkens, Handelns und 
Fühlens. "Und jetzt, sagt Rosalind, werde ich mit dem Kopf durch die Wand gehen. » (130) Sie imaginiert ihre Ein- und Ausbrüche, die von der Umwelt als Verrücktheiten taxiert werden und sie - oder ist es Martha ? - in ein Gefängnis bringen, das auch eine psychiatrische Abteilung sein könnte : eine weiß gestrichene Zelle mit wenigen Möbeln und einem schmalen Bett. Sie riskiert Polizeiverhöre und soziales Außenseitertum, wenn sie es wagt, sich auf die gesellschaftlich nicht akzeptierten Aspekte und Facetten ihres individuellen Seins einzulassen, wenn sie ihre Grenzen sprengenden, Grenzen transgredierenden, über- und unterschreitenden Fähigkeiten inszeniert oder auch bloß anvisiert. Dann wird der Bahnhof zur wichtigen Metapher individuellen Selbstverständnisses, der die Fähigkeit des Weggehen-Könnens - sei es nach Spanien, nach Amerika oder nach Grönland - illustriert; denn : "Wer bleibt, hat den Kampf gegen seine Natur gewonnen " (78), heißt es. Martha und Clairchen fungieren als Modelle verdrängter, vergessener Lebensmöglickeiten, die die Reduktion auf die rationalen Potenzen des modernen Menschen als armselige Verkümmerungen erscheinen lassen, sie figurieren als Prototypen des Irrationalen, die die Unvernunft sei es ihres Körpers, sei es ihrer seelischen Bedürfnisse in unkonventionellen, das Kriminelle wie das Verrückte ständig streifenden Weisen zu leben versuchen und damit jeden Begriff einer « Ordnung » ad absurdum führen.

«In Clairchen muß ein anderes, wilderes Blut gelebt haben, und auch in Martha, eins, das sich auflehnen mußte gegen die Dressur, das in den Kopf stieg, daß der fürchtete zu zerplatzen wie eine überreife Melone und darüber alle anderen Ängste vergaß [...]. » (116)

12 Allerdings - und dies scheint der Preis moderner Sozialitäten zu sein - gelingt ein Zusammendenken rationaler, seelischer und körperlicher Fähigkeiten und Bedürfnisse in der Moderne nicht mehr. Wir wissen, was wir verloren haben, was uns abhanden gekommen ist: die Ganzheit der Person und ihrer Autonomie, aber die Wiederherstellung einer wenn auch nur provisorischen Ganzheit lässt sich nur auf dem Weg des Fragments, der Collage und der Montage bewerkstelligen. Ein künstliches Spektakel, zusammengefügt aus den Bestandteilen traditioneller Topoi literarischer Figurendarstellung, ist der schwache Ersatz für ein verloren gegangenes identisches Ich. So sehr der Text Die Überläuferin die Austauschbarkeit seiner drei weiblichen Figuren verdeutlicht - wenn etwa gar innerhalb eines Satzes Martha und Rosalind ihre Plätze austauschen und ineinander « überlaufen »-, so klar unterscheidbar bleiben sie doch als verschiedene Entwürfe weiblichen Seins, die sich nicht mehr zu einem Ganzen zusammenfügen lassen. Rosalind mag auch Martha sein und sich in Clairchen hineinfinden, ein neues Ich, das aus den dreien sich neu zusammensetzte, gibt es nicht mehr - auch nicht im fiktionalen Bereich der Literatur. So dass die entworfenen Frauenfiguren wohl zeigen, was wir verloren haben und was uns fehlt, aber nicht wirklich eine neue Utopie ganzheitlichen Seins zu kreieren imstande sind.

4.

13 Ein solches Bemühen um Re-evokation verloren gegangener Potenzen und einer «Lebensfülle », die nur ex negativo, d.h. nur im Bewusstsein ihrer notwendigerweise defizitären Rekonstruktion gedacht und erprobt $\mathrm{zu}$ werden vermag, ohne auf Realisierung hoffen zu können, erfordert neue Schreibweisen, die in der Abwehr jener ästhetischen Postulate, die dem Realismus-Konzept verpflichtet sind und auf die Wiedererkennbarkeit von Fakten Wert legen, dem Bereich des Traums, des 
Unbewussten und des Intuitiven Raum lassen und die von den Surrealisten des frühen 20. Jahrhunderts auf vorbildhafte Weise vorgestellt und vorgeprägt worden sind. Unübersehbar ist, wie sehr Monika Marons Roman «Die Überläuferin» die Tradition surrealistischen Schreibens in seinem das Leben wie das Denken revolutionierenden Impuls weiterschreibt - nicht zufällig trägt Marons Martha eindeutig Züge von Bretons Nadja $^{11}$-, wie sie in dem Vertrauen auf jene "Logik des Wunderbaren", die die surrealistischen Autoren der 20er Jahre gegen die Dominanz des Rationalen setzten oder zu setzen beanspruchten, sich sowohl den "Traumbericht» ("récit de rêves") wie das " automatische Schreiben» (« écriture automatique ») zu eigen macht; jene Formen «neuen » Schreibens, die nach Breton als besonders geeignet gelten müssen, um das «Diktat des Denkstromes» und die «Ausschaltung jeglicher Vernunftkontrolle » zu erreichen ${ }^{12}$.

Sie inszeniert die Auflösung konventioneller Erzähltraditionen - auch hierin dem Diskurs des Irrsinnigen verpflichtet -, um jene aus dem Diskurs rationalen Erzählens ausgestoßenen Bereiche des Traums, der Vision, der Imagination, des Wahnsinns wieder in den Erzählstrom hereinzuholen. Nicht unter bewährtem Rückgriff auf die anerkannten Formen des Märchens oder der prophetischen Rede, sondern als disparates Konglomerat unzusammenhängender Erinnerungsfetzen, Traumsplitter, Visionsschübe, das - und hierin unterscheidet es sich fundamental von dem Breton'schen Kokettieren mit dem Spiel des Zufälligen und Nicht-Berechenbaren nicht der faszinierenden Inszenierung weiblicher Unvernunft und Phantasie das rationale, männliche Autor-Ego entgegensetzt ${ }^{13}$, sondern sowohl die Erzählerin wie die Autorin selbst als Teilnehmende an diesem Dissoziationsprozess in den auflösenden Erzählvorgang integriert.

Denn nicht als eine souveräne, rationale Autor-Instanz, die die Geschichte oder NichtGeschichte bestimmt und die - wie Foucault sagt - « der beunruhigenden Sprache der Fiktion ihre Einheiten, ihren Zusammenhang, ihre Einfügung in das Wirkliche gibt ${ }^{14}$, setzt sich hier die Autorin in Szene, sondern sie splittet sich auf in die Vielheit von Erzähler-Figuren und erzählten Figuren, die ihrerseits fiktive Figuren imaginieren. Kein stabiles, Geschichte und Fortgang der Handlung legitimierendes Autor-Ich ist hier auszumachen, sondern ein Geflecht aus verschiedenen Erzählinstanzen, aus deren Perspektiven gleichermaßen geredet wird wie über sie geredet wird. Nicht einmal der Blickwinkel, aus dem die Geschichte erzählt wird, ist ein verlässlicher, gleich bleibender. Mal ist es ein Ich, das spricht, mal Rosalind, mal Martha. Und dann noch sind es die Figuren des Zimmertheaters, die sich einmischen und ihren Platz in der Vielheit der Diskurse beanspruchen, die einfach auch mitreden wollen. Was entsteht, ist ein changierendes Kaleidoskop divergierender Erzählmöglichkeiten, das die Legitimationen des Erzählens ebenso betrifft wie die Erzählhaltungen und die Erzählmodi. Wenn die uneingeschränkte Autorität eines rationalen Autor-Konzepts nicht länger unhinterfragt gelten soll, dann kann auch das Primat eines monoperspektivischen, einstromförmigen Erzählens nicht länger aufrechterhalten werden. Das Was wie das Wie des Erzählens stehen gleichermaßen zur Disposition. Weder wird beansprucht, die Wahrheit über die Wirklichkeit oder auch nur die Wirklichkeit zu beschreiben - im Gegenteil : Rosalind bestärkt ihre Empfindung, immer «in zwei verschiedenen Welten" (94) gelebt zu haben, d.h. die Erfahrung einer einheitlichen, zusammenhängenden Welt erst gar nicht zu kennen -, noch ist in der Art der Darstellung ein einheitliches Prinzip auszumachen: Erinnerungsarbeit, 
Traumprotokoll, fiktives Zimmertheater, Dialogszenen und Angstvisionen lösen einander ab, gehen oft bruchlos ineinander über.

Der Verzicht auf logisch-stringentes Erzählen, das auf eine Pointe gerichtet ist, das Zusammenhang und Plausibilität suggerieren will, versteht sich somit auch als Antwort auf oder gegen männliche Erzähl- und Autorkonzepte, die die Wirksamkeit einer Erzählung an die rationale Erzählkompetenz ihres Autors rückbinden und weibliche Autorinnen vor die Wahl stellen, entweder rationale, männliche Autor-Vorstellungen fortzuführen oder aber - wofür der vorliegende Text ein Beispiel sein kann - auf andere, weniger rationale, d.h. aber auch weniger gut beleumdete Autorkonzepte und daraus resultierende Erzählmöglichkeiten zu rekurrieren, die, indem sie die Instanz des Autors/der Autorin in einem Geflecht austauschbarer Beziehungen von erzählenden und erzählten Figuren verschwinden machen, die Möglichkeit eines anderen, weiblichen Autorbegriffs ${ }^{15}$ erahnen lassen, der sich nicht als großer Zampano in Szene setzt, sondern sich als Konstrukt möglicher Erzählhaltungen zu erkennen gibt und das Prinzip der Fiktionalität des Erzählens auch vor sich selbst nicht aufgibt.

Die Gefährlichkeit eines solchen (weiblichen) Erzählens geht weit über die feministische Provokation hinaus. In einer der schaurigsten Szenen des Romans «Die Überläuferin » soll Martha - oder Rosalind oder aber das Ich, als dessen Projektionen Rosalind wie Martha gleichermaßen gelten können ${ }^{16}$ - zu Tode gebracht werden ; und zwar nicht zufälligerweise aufgrund ihres Schreibens. Sie wird von einem « führenden Mitglied der Assoziation dichtender Männer » (155) aufgesucht, das ihr die Verbrechen ihres Schreibens wie die Notwendigkeit ihrer Elimination nahezubringen versucht. Da ihre "Schuld» darin liege, "schlechte», nämlich aus weiblicher Sicht und aus weiblichen Anliegen formulierte Texte zu schreiben, könne diese auch nicht durch den Hinweis auf «schlechte » männliche Literatur gemildert werden. Texte von Männern, auch «schlechte» Texte von Männern würden es nicht vermögen, das alte, traditionelle System abendländischen Wissens und Schreibens in Gefahr zu bringen, wie dies Texte von Frauen tun würden, deren Qualitäten den anerkannten Kriterien europäisch-rational-männlichen Denkens und Schreibens zutiefst widersprächen. Um die Tradition des mühsam über die Jahrtausende aufgebauten diskursiven Systems zu gewährleisten, müssten gravierende Infragestellungen und Angriffe - wie jene schreibender Frauen - abgewehrt, ja verhindert werden. "Was ", so die entrüstete Frage des Abgesandten, "glaubst du, würde geschehen, wollte man plötzlich die nächste Schicht bauen zum einen Teil wie bisher, zum anderen Teil aus Wind, Sonnenstrahlen und Wellenschaum.» (159) Das, was das Schreiben von Frauen kennzeichne und was durchaus auch als Selbstbeschreibung des Romans "Die Überläuferin» gelten könnte: nämlich «Romantizismen, Lyrismen, Pathos, Selbstmitleid, Infantilismus und modisches Feministengeplapper » (156), ist nicht nur das Inkompatible, das ganz Andere, es ist vor allem auch dies, was die Ordnung des Akzeptierten, die Normen des Selbstverständlichen außer Kraft setzt, was nicht nur eine «andere» Existenz - gemäß dem surrealistischen Slogan "L'existence est ailleurs $»^{17}$ - anvisiert, sondern vor allem auch diese, hier und jetzt vorhandene, ganz konkrete Existenz (in ihren lebenspraktischen, vor allem aber auch in ihren diskursiven und machtstrategischen Aspekten) aus den Angeln hebt.

Es versteht sich, dass dieses Unterfangen die Nähe des Wahnsinns riskiert und vom männlichen Beobachter als « Hysterie » (160) abgetan wird; es versteht sich auch, dass Martha letztlich dem Attentat nicht entkommt und ermordet wird - von jenem zu 
einem Vampir sich verwandelnden Abgesandten, in dessen Namen «Heinrich» die Ambivalenz Marons eigener Lektüreerfahrungen, d.h. die Zwiespältigkeit ihrer eigenen zwischen Bewunderung (für Heinrich Heine ${ }^{18}$ ) und skeptischer Distanz (gegenüber Heinrich von Kleist ${ }^{19}$ ) oszillierenden Leseerfahrungen mit Texten männlicher Autoren mitschwingt. Ein Ausweg ist nicht wirklich in Sicht.

\section{5.}

Aber kann es, so wäre abschließend zu fragen, wenn alle Grenzen systematisch in Frage gestellt, überschritten, umgestoßen werden, überhaupt darum gehen, « Auswege » zu finden, so etwas wie "richtige » oder auch nur «praktikable » Wege auszuprobieren, nachzuzeichnen, vorzuschlagen, oder liegt der Reiz des hier vorgestellten Buches nicht darin, uns dazu zu ermuntern, einfach aufzubrechen, ohne den Weg bereits zu kennen und uns nicht dabei schon von vornherein mit unserer Angst, als Wahnsinnige, als Irrsinnige oder ganz Verrückte abgestempelt zu werden, selbst im Weg zu stehen!?

Was ist dieses Buch anderes als eine große Revision unserer Irrtümer und falschen Überzeugungen, eine halb naive, halb irrsinnige Abrechnung mit unseren Scheinidentitäten und sozialen Ansprüchen - sei es auf Respektabilität, auf Geliebtwerden-Wollen, auf Karriere oder Anerkennung - und ein großes Plädoyer für die Besinnung auf die Potenzen unserer Imagination und Phantasterei, die die Nähe des Wahnsinns riskiert, die sich aber von dieser - übrigens immer nur von den anderen zugeschriebenen - Wahnsinnsnähe nicht verunsichern lässt, sondern das Feuerwerk ihrer Inspiration, ihrer Originalität und ihrer Dynamik entfachen sollte.

21 Es geht nicht um Wahrheit und es geht auch nicht « wirklich» um die Wirklichkeit. Es geht um die Literatur als Ort des Möglichen und Unmöglichen, wo die Grenzen zwischen Wahrheit und Lüge - die von Martha so oft willkürlich ignoriert werden verschwimmen, weil sie hier nicht von Belang sind. Es geht um die Einforderung von im wahrsten Sinn des Wortes ästhetischen Qualitäten als da sind: Fiktionalität und Vieldeutigkeit ${ }^{20}$. Literatur kann und muss - will sie nicht ihre ureigenste Legitimation aufs Spiel setzen - ihre Nähe zu Wahnsinn und Verbrechen kultivieren, und sie kann es, weil sie letztlich doch nie mehr ist und sein kann als bloßer "Schein" - oder wie Marthas Professor in seiner Abrechnung mit dem zeitgenössischen Theater des realen Sozialismus, dem Zeitstück, so treffend formuliert : «Denn was, außer Illusion, sollte das Theater sein [...]»(139). Dass uns aus der Konfrontation mit einem ebenso radikalen wie letztlich unwirklichen Szenario hemmungsloser Phantasie der Mut zu unserer eigenen Imaginationskraft erwachsen möge, darin liegt einer der utopischen Entwürfe des Romans - und sei es auch nur im hoffnungsfrohen Modus des Konjunktivs, mit dem der Roman endet: «Den Mund weit öffnen und das Wasser in mich hineinlaufen lassen, naß werden, dachte sie, vom Regen naß werden, ja, das wäre schön. » (221) 


\section{NOTES}

1. Monika Maron : Die Überläuferin, Frankfurt/Main : Fischer 1988. (In der Folge mit einfacher Seitenzahl zitiert.)

2. Vgl. Bettina Rabelhofer : "Die qualitative Grenzziehung zwischen dem ,Normalen` und dem ,Verrückten', dem ,Gesunden ' und dem ,Kranken', dem ,Schönen` und dem ,Häßlichen` zeugt von einem ,Ordnungswillen', der die Sprache des vernünftigen Diskurses mit der Macht zum Ausschluß des Devianten ausstattet. «Bettina Rabelhofer: «Des Dichters Aug in schönem Wahnsinn rollend». Zum Verhältnis von Psychopathologie und Ästhetik um 1900 », in : Alice Bolterauer, Elfriede Wiltschnigg (Hg.) : Kunstgrenzen. Funktionsräume der Ästhetik in Moderne und Postmoderne, Wien: Pasagen 2001, S.127-141, 127. Rabelhofer argumentiert hier unter Bezugnahme auf Foucault, für den der «Wahnsinnige» derjenige ist, « dessen Diskurs nicht ebenso zirkulieren kann wie der der anderen : sein Wort gilt für null und nichtig, es hat weder Wahrheit noch Bedeutung [...]. « Michel Foucault : Die Ordnung des Diskurses. Inauguralvorlesung am Collège de France (2. Dez. 1970), Frankfurt/Main, Berlin, Wien 1977, S. 8.

3. «Die gesellschaftliche Zuweisung spezifischer Orte und Terrains setzt einen Standard von Normalität voraus. Wie klar ist dieser heute noch? «Wolfgang Welsch : «Identität im Übergang », in : ders. : Ästhetisches Denken, Stuttgart : Reclam 1995, S. 168-200, 170.

4. Wolfgang Beutin [u.a.]: Deutsche Literaturgeschichte: von den Anfängen bis zur Gegenwart, Stuttgart, Weimar : Metzler 2001, 560f.

5. Ebd., S. 561.

6. Monika Maron : Flugasche, Frankfurt/Main : Fischer 1981, S. 201.

7. Ebd., S. 72.

8. Vgl. Hans-Jürgen Schmitt (Hg.) : Die Expressionismusdebatte. Materialien zu einer marxistischen Realismuskonzeption, Frankfurt/Main : Suhrkamp 1976.

9. Monika Maron : Animal triste, Frankfurt/Main : Fischer 1996, S. 239.

10. Vgl. Hartmut und Gernot Böhme: Das Andere der Vernunft. Zur Entwicklung von Rationalitätsstrukturen am Beispiel Kants, Frankfurt/Main : Suhrkamp 1992.

11. Wenn es von Nadja heißt : «J'ai pris, du premier au dernier jour, Nadja pour un génie libre, quelque chose comme un de ses esprits de l'air que certaines pratiques de magie permettent momentanément de s'attacher, mais qu'il ne saurait être question de se soumettre ", dann wäre diese Beschreibung beinahe 1:1 auch auf Martha übertragbar. André Breton: Nadja, Paris: Gallimard 1981, S. 130.

12. Der Surrealismus sei ein : "Automatisme psychique pur par lequel on se propose d'exprimer, soit verbalement, soit par écrit, soit de toute autre manière, le fonctionnement réel de la pensée. Dictée de la pensée, en l'absence de tout contrôle exercé par la raison, en dehors de toute préoccupation esthétique ou morale.» André Breton : «Manifeste du surréalisme », in : ders. : Manifestes du surréalisme, Paris : Gallimard 1981, S.11-64, 37.

13. Der Beobachterstatus des männlichen Autors wird deutlich hervorgehoben : «Il me semble que je l'observe trop, comment faire autrement ?» Breton, Nadja, S. 104.

14. Foucault, Die Ordnung des Diskurses, S. 20.

15. Vgl. dazu : Peggy Kamuf : «Writing like a Woman », in : S. McConnell-Ginet u.a. (Hg.) : Women and Language in Literature and Society, New York: Praeger 1980, S. 284-299; Genia Schulz: "Anmerkungen zum Verschwinden des Autors und zum Erscheinen der Autorin», in: Inge Stephan, Sigrid Weigel, Kerstin Wilhelms (Hg.) : «Wen kümmert's, wer spricht.» Zur Literatur- und Kulturgeschichte von Frauen aus Ost und West, Köln, Wien 1991, S. 57-62.

16. «Martha setzt sich wieder auf den Stuhl, mit dem Rücken zu mir, was in mir die Vorstellung auslöst, ich stünde mir selbst im Rücken [...]». (153) 
17. So lautet der letzte Satz von Bretons erstem surrealistischen Manifest. Breton, Manifeste du surréalisme, S. 64

18. Vgl. dazu Monika Marons Heine-Aufsatz: Monika Maron : «Liebster Heinrich !», in : dies. : Nach Maßgabe meiner Begreifungskraft. Artikel und Essays, Frankfurt/Main : Fischer 1995, S. 57-59.

19. Vgl. dazu Monika Marons Rede anlässlich der Verleihung des Kleist-Preises : Monika Maron : «Nach Maßgabe meiner Begreifungskraft», in: dies.: Nach Maßgabe meiner Begreifungskraft. Artikel und Essays, Frankfurt/Main : Fischer 1995, S. 103-111.

20. Vgl. dazu die Ausführungen von Peter Brenner : «Was ist Literatur? », in : Renate Glaser, Matthias Luserke (Hg.) : Literaturwissenschaft - Kulturwissenschaft. Positionen, Themen, Perspektiven, Opladen: Westdeutscher Verlag 1996, S. 11-47. Brenner nennt "Fiktionalität», «Vieldeutigkeit» und «Schönheit» als zentrale Prinzipien der Diskussion über Kunst und Literatur.

\section{RÉSUMÉS}

Monika Marons Roman Die Überläuferin versteht sich als Plädoyer gegen die Einseitigkeit eines rationellen Denkens, das sowohl unser individuell-privates Dasein prägt wie es den politischen Diskurs und die Tradition des Erzählens bestimmt. Dagegen setzt Monika Maron die Fülle der Imagination und Phantasie, die die Nähe des Wahnsinns und Verbrechens nicht scheut und die in ihrer Sprengkraft als ebenso utopisches wie gefährliches Element erscheint.

Le roman Die Überläuferin / La transfuge de Monika Maron s'inscrit dans la tradition d'une écriture surréaliste et plaide pour un être plus rond, plus complet qui intègrerait outre notre raison, également nos compétences spirituelles et naturelles voire biologiques. Un tel projet risque de se heurter au discours dominant, et d'être taxé de «fou », d'« hystérique ", mais - comme le dit André Breton dans le «Manifeste du surréalisme »: «Ce n'est pas la crainte de la folie qui nous forcera à laisser en berne le drapeau de l'imagination.»

\section{AUTEUR}

\section{ALICE BOLTERAUER}

Graz 